\title{
Smart Stylet: The Development and Use of a Bedside External Ventricular Drain Image-Guidance System
}

\author{
Vaibhav Patila Rajiv Guptac Raúl San José Estépar ${ }^{\mathrm{a}}$ Ronilda Lacson ${ }^{\mathrm{a}}$ \\ Arnold Cheung ${ }^{c}$ Judith M. Wong ${ }^{b}$ A. John Popp ${ }^{b}$ Alexandra Golby ${ }^{a} b$ \\ Christopher Ogilvy ${ }^{d}$ Kirby G. Vosburgh ${ }^{a}$ \\ Departments of a Radiology and ${ }^{\mathrm{b}}$ Neurosurgery, Brigham and Women's Hospital, 'Department of Radiology, \\ Massachusetts General Hospital, and d Department of Neurosurgery, Beth Israel Deaconness Medical Center, \\ Boston, Mass., USA
}

\author{
Key Words \\ Ventriculostomy · Image-guided surgery - Electromagnetic \\ concepts $\cdot$ Neuronavigation
}

\begin{abstract}
Background: Placement accuracy of ventriculostomy catheters is reported in a wide and variable range. Development of an efficient image-guidance system may improve physician performance and patient safety. Objective: We evaluate the prototype of Smart Stylet, a new electromagnetic image-guidance system for use during bedside ventriculostomy. Methods: Accuracy of the Smart Stylet system was assessed. System operators were evaluated for their ability to successfully target the ipsilateral frontal horn in a phantom model. Results: Target registration error across 15 intracranial targets ranged from 1.3 to $4.6 \mathrm{~mm}$ (mean $3.1 \mathrm{~mm}$ ). Using Smart Stylet guidance, a test operator successfully passed a ventriculostomy catheter to a shifted ipsilateral frontal horn 20/20 (100\%) times from the frontal approach in a skull phantom. Without Smart Stylet guidance, the operator was successful $4 / 10(40 \%)$ times from the right frontal approach and $6 / 10(60 \%)$ times from
\end{abstract}

the left frontal approach. In a separate experiment, resident operators were successful 2/4 (50\%) times when targeting the shifted ipsilateral frontal horn with Smart Stylet guidance and 0/4 (0\%) times without image guidance using a skull phantom. Conclusions: Smart Stylet may improve the ability to successfully target the ventricles during frontal ventriculostomy.

c) 2015 S. Karger AG, Basel

\section{Introduction}

Radiologic images are often presented to help surgeons navigate and target during procedures. Image guidance has been used to effectively guide many neurosurgical procedures but is not widely used for ventriculostomy due to the time required for instrument setup, cost, and complexity of use. A recent nationwide poll of neurosurgeons found that greater than $50 \%$ of respondents would use an image-guidance system that guarantees placement $100 \%$ of the time if it can be implemented within $10 \mathrm{~min}$ [1]. Attempts to use fixtures or guides have not been shown to significantly improve placement accuracy over

\section{KARGER 125}

(c) 2015 S. Karger AG, Base

$1011-6125 / 15 / 0931-0050 \$ 39.50 / 0$

E-Mail karger@karger.com

www.karger.com/sfn
Vaibhav Patil, MD, MS

Brigham and Women's Hospital, Harvard Medical School

75 Francis Street, ASBI L1-050

Boston, MA 02115 (USA)

E-Mail patil@bwh.harvard.edu 
Fig. 1. Smart Stylet system component

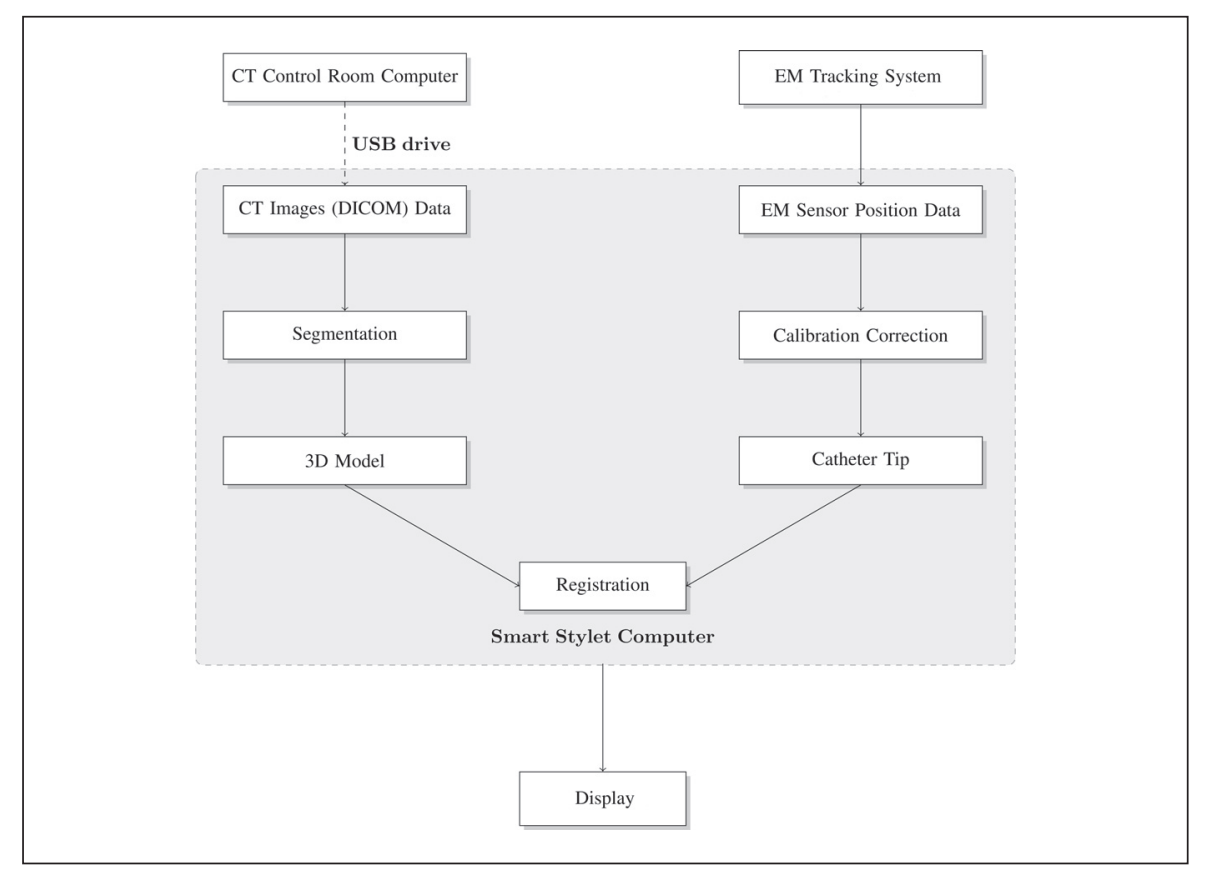

the freehand technique [2]. Recent improvements in image-guidance technology, such as portable components and high-performance computing, offer prospects for the implementation of more sophisticated systems that will fit within the constraints of clinical practice.

Reported rates of appropriate intracranial catheter placement range from less than $50 \%$ to greater than $90 \%$ [3-11]. Unsuccessful placement of the cannula requires its withdrawal and an additional pass of the ventriculostomy catheter. Greenberg's Handbook of Neurosurgery [12] dictates a limit of three attempts for the operator to successfully target the ventricle. The same survey referenced above reports a wider range of clinical practice with $46 \%$ of respondents having witnessed five or fewer passes [1]. Associations between multiple attempts to successfully cannulate the ventricle and intracranial hemorrhage have not been thoroughly studied [13]. Properly placed catheters malfunction less commonly and require less frequent replacement to maintain patent flow of cerebrospinal fluid [14]. Catheter replacement has been significantly associated with ventriculostomyrelated infection [15].

In this article, we present the development of Smart Stylet as a response to the need for improved catheter placement accuracy. This report presents initial measurements of performance for the prototype Smart Stylet system. We first established baseline expectations for system and operator error. We then requested neurosur- gical resident operators to target the ipsilateral frontal horn of the ventricular system in a phantom model using Smart Stylet.

\section{Methods}

\section{Smart Stylet Components}

An electromagnetic (EM) tracking system, a conventional ventriculostomy stylet and catheter, and a Dell (Round Rock, Tex., USA) Precision personal computer with display were used to assemble the Smart Stylet system (fig. 1). All components are suitable for clinical use and commercially available for a total cost of approximately USD 20,000. We used a pulsed direct current EM tracking system designed and marketed by Ascension Technologies (Shelburne, Vt., USA). A prototype portable flat plate consisting of three EM transmitters (fig. 2) provided the local EM field where the position and orientation of the EM tracker coils could be interrogated by the system. The plate was designed to be under the patient's head on a hospital bed and composed of a proprietary material to protect the EM field from ferromagnetic objects below the transmitter.

Smart Stylet's software module was implemented in 3D Slicer 2.7 , open-access research software for image analysis and imageguided therapy (www.slicer.org). The software interface consists of a three-dimensional segmented display, two reformatted computed tomography (CT) displays, and three traditional radiology CT imaging views. All imaging displays were dynamically viewed using coordinates transmitted from the EM system. Within the segmented display, two trajectories were defined - one along the path of the stylet (incident) and the other an ideal straight-line trajectory from the tip of the catheter to the target (planned). The 
Fig. 2. Prototype flat plate EM transmitter. The transmitter can be positioned and removed from under the patient's head at the bedside. Three small cube transmitters are positioned on a plate to create a working volume around the patient's head.

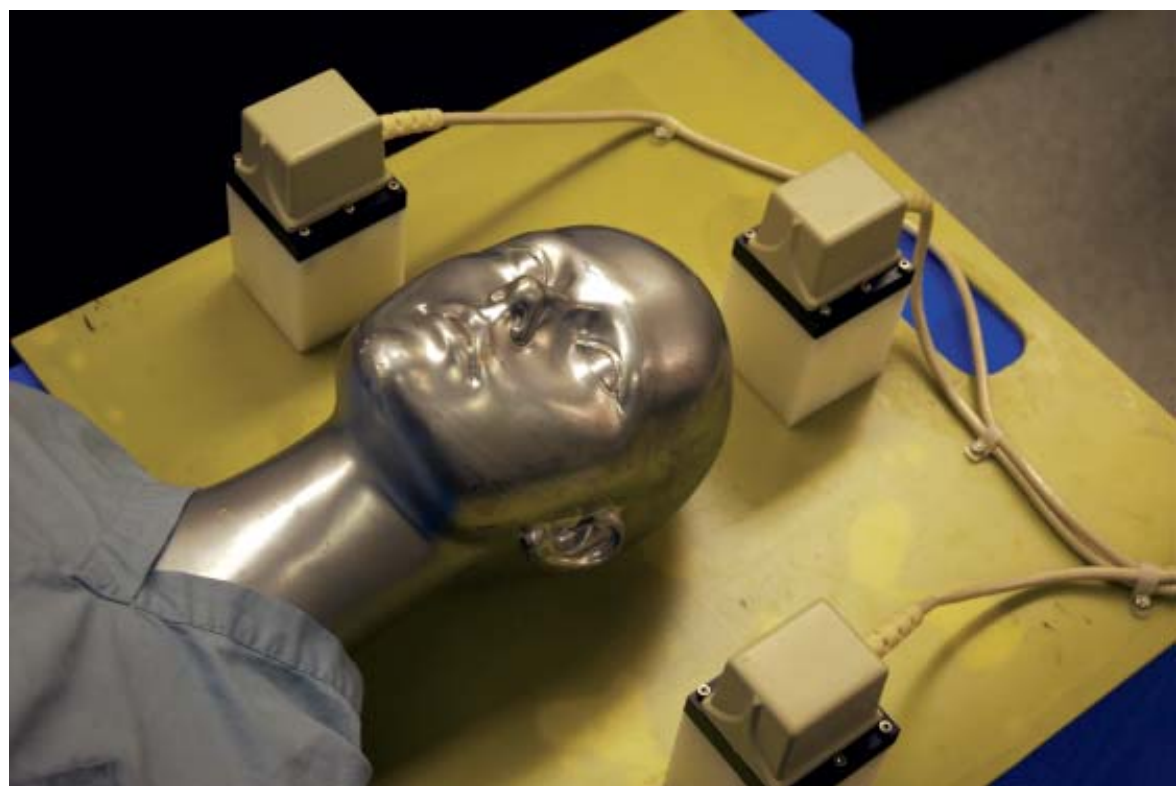

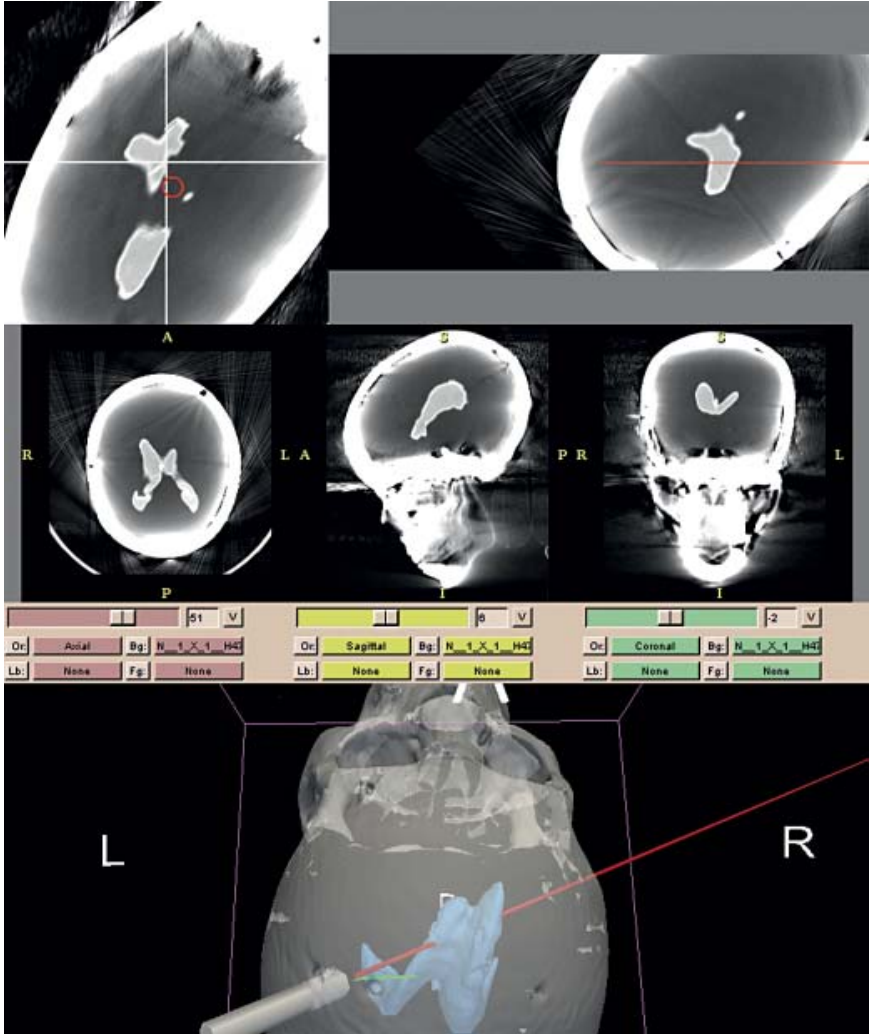

Fig. 3. Smart Stylet display. Above left: bull's eye reformat (axial). Above right: trajectory reformat (coronal). Below: segmented view (green $=$ planned trajectory, red $=$ incident trajectory). three-dimensional display was designed to simulate the operator's environment at the head of the patient bed and help provide orientation during navigation (fig. 3, below display). The simulation displays the stylet in relation to surrounding anatomy at 20 frames per second. Oblique axial slices (fig. 3 , above left) display dynamically at an angle perpendicular to the stylet trajectory and in the plane of the target ventricular system. The image frame was designed to help the operator align incident and planned trajectories. The stylet's incident trajectory was defined as a red circle that translates across the oblique axial slice view. The origin of the $\mathrm{x}$ and $y$-axes represent the planned trajectory. During the procedure, the operator maintains the red circle directly over the origin to achieve a 'bull's eye' effect. Oblique coronal slices were positioned along the incident trajectory of the stylet (fig. 3, above right). The view was designed to help the operator align catheter trajectory with the target ventricular system. The red line represents the incident trajectory of the stylet and remains stationary in the image frame. Figure 3 (above right) shows a portion of the red trajectory line at the inner plate of the skull phantom and represents the stylet tip - the stylet is not shown. During the procedure, the operator aligns the incident trajectory with patient ventricular anatomy.

In the 'Accuracy' experiment described below, a 1.8- $\mathrm{mm} 6$ degrees of freedom sensor was used to derive the position and orientation of a rigid needle pointer. In the 'Bench' and 'User' experiments, a prototype $0.3-\mathrm{mm} 5$ degrees of freedom sensor was used to determine the position and orientation of the catheter and stylet. We did not find targeting to require the measurement of angular rotation along the length of the stylet. We selected a $0.3-\mathrm{mm}$ sensor because it was small enough to be placed at the tip of the stylet within the catheter's lumen (fig. 4) and thereby minimized calibration errors.

\section{Test Phantoms}

Accuracy Phantom

A test Accuracy Phantom (not shown) was built using a plastic skull replica for use in the Accuracy experiment. Five CT fiducial 


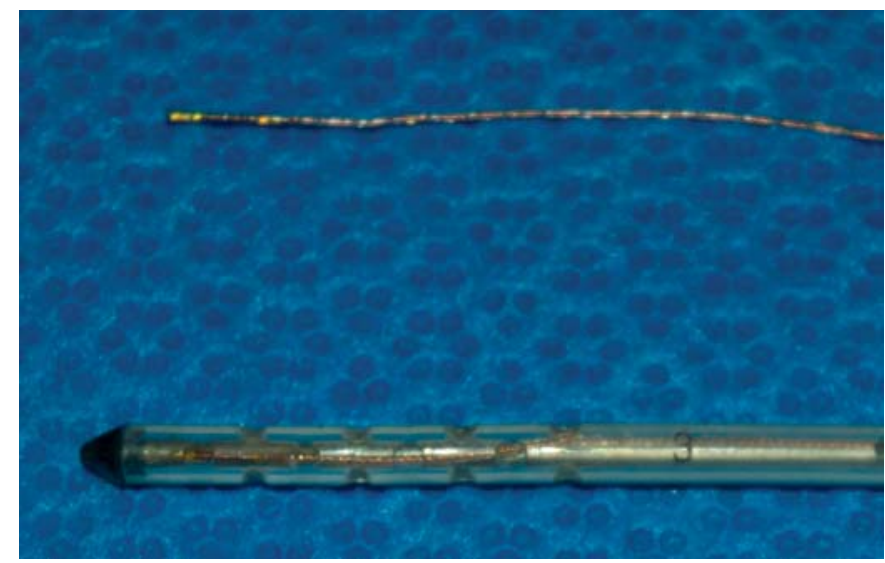

Fig. 4. Prototype $0.3-\mathrm{mm}$ EM sensor (above) in comparison to an EVD catheter (below). An EM sensor is also shown in the catheter alongside the stylet.

markers were placed across the frontal and temporal regions of the phantom for registration. Fifteen intracranial CT fiducial targets were placed on the base of the empty cranial vault along the frontal, sphenoid, temporal and occipital bones.

\section{Procedure Phantom}

The above test phantom was modified to include a model of a shifted ventricular system for use in the Bench and User experiments (Procedure Phantom, segmented image shown in fig. 3). To build the phantom, a patient CT image data set with significant midline shift was de-identified. The image was processed using a combination of thresholding, morphological operations and manual outlining to make a segmented model of the ventricles. The model was converted for use by computer-aided design software and a three-dimensional printer processed the image to create a physical ventricular model. The ventricular model was rigidly fixed within the plastic skull's cranial vault using the approximate sagittal planes of the medial canthi and approximate coronal plane of bilateral tragi.

To create entry points for the Bench and User experiments, three 0.25 -inch frontal twist drill holes were created on the skull replica, two left frontal and one right frontal. Ten centimeters were measured posterior to the nasion along the midline. Three centimeters lateral from the midline were measured in both directions and two twist drill holes were made using a hand crank drill - one left and one right. Once it was determined that the left frontal horn was difficult to access from the traditional twist drill hole coordinates, 2 more centimeters were measured lateral from the left hole and an additional entry point was placed. In all instances, the hand crank drill bit was positioned perpendicular to the plane tangential to the entry point. It was manually confirmed that the target ventricle could be reached from all entry points at a catheter depth no greater than $7 \mathrm{~cm}$.

\section{Accuracy Measurement Experiment}

The aim of the Accuracy measurement ('Accuracy') experiment was to measure the baseline accuracy of the Smart Stylet system. First, a segmented three-dimensional model of the Accuracy
Phantom was created. The Accuracy Phantom was imaged in a Siemens Somatom CT scanner using $1.25 \mathrm{~mm}$ slice thickness. Images were segmented using Slicer 2.7 software.

\section{Calibration and Registration}

The rigid needle was calibrated to define the location of the needle tip in relation to the attached $1.8-\mathrm{mm}$ sensor [16].

The Accuracy Phantom was placed in the transmitter space. The calibrated needle was then used to acquire spatial coordinates of the three and five external fiducial markers on the frontal and temporal regions of the Accuracy Phantom in two separate acquisitions of data. A transformation to convert the registration points on the phantom to their coordinates with respect to the CT scan was automatically derived [17], defining a registration matrix for three fiducials and another for five fiducials. Each registration matrix was used to view the calibrated stylet with respect to the CT image.

\section{Data Gathering}

Five successive acquisitions of data were completed to determine spatial coordinates of the intracranial target points using the tracked rigid needle. The tip of the tracked needle was placed on each of the 15 intracranial targets at the base of the cranial vault in the Accuracy Phantom. Fifteen sensor readings were acquired. The experiment was done twice - once with a 3-point registration and once with a 5-point registration.

\section{Data Analysis}

All data analysis was carried out using Matlab 7.4.0 (The Mathworks, Inc., Natick, Mass., USA). For this experiment, target registration error (TRE) was defined as the Euclidean distance between the calibrated, registered sensor readings and the positions of corresponding intracranial targets on CT imaging. TRE was calculated for each of the 15 intracranial target points and averaged across five sets of data per registration matrix.

\section{Bench Level Testing}

The aim of the Bench level testing ('Bench') experiment was to evaluate the performance of Smart Stylet with an initial user. The user was a physician with entry-level clinical ventriculostomy experience and a partial developer of the Smart Stylet system. The Procedure Phantom containing the shifted ventricular model was imaged and segmented.

\section{Calibration and Registration}

First, the stylet was calibrated to define the location of the catheter tip with respect to the attached 0.3-mm EM sensor. The Procedure Phantom was placed in the transmitter space. A separate EM sensor was used to collect a surface map of points by tracing the calvarium with adequate coverage of frontal, temporal and occipital areas. The collection of points was used to register the surface of the skull phantom to that of the segmented model [18].

\section{Ventriculostomy Catheter Placement}

The test operator inserted the stylet 10 times without image guidance and 10 times with image guidance. Each pass of the catheter was made to a maximum depth of $7 \mathrm{~cm}$ or if the operator encountered a solid structure within the phantom. The EM tracking system was used to acquire spatial coordinates of the catheter tip at the completion of each pass. On each attempt, the operator was 
blinded to success or failure when targeting the ventricle. Each attempt was separated by at least $1 \mathrm{~h}$ to minimize visual-spatial learning.

\section{Data Analysis}

The data were analyzed using a method to evaluate catheter trajectory. Success or failure was postexperimentally defined by visualizing the trajectory with respect to the segmented model of the ventricular system. A trajectory beyond the final location of the catheter tip was extrapolated using points representative of the twist drill hole and Smart Stylet's final location on each pass of the catheter. A 'hit' or 'miss' was recorded dependent on intersection of the extended trajectory with the ventricular model.

\section{User Evaluation}

The aim of the User evaluation ('User') experiment was to evaluate Smart Stylet when used by neurosurgical residents in a clinical simulation representative of distorted ventricular anatomy. Under approval of the Institutional Review Board, two resident physicians from the neurosurgical service were randomly recruited to test and evaluate Smart Stylet.

The Smart Stylet system was implemented as in the Bench experiment and tested on the Procedure Phantom that housed a shifted ventricular model. The same registration and calibration transformation matrices used in the Bench experiments were maintained in this series of experiments for ease of comparison. Each resident passed the catheter once with and once without guidance from both frontal approaches for a total of four passes per resident operator. Data acquisition with and without Smart Stylet guidance were separated by a minimum of $1 \mathrm{~h}$ to minimize visual-spatial learning in the study subjects. The residents were requested to pass the catheter to a maximum depth of $7 \mathrm{~cm}$ or until solid surface was encountered within the cranial vault.

\section{Results}

\section{Accuracy Measurement Experiment}

TRE ranged from 1.5 to $4.6 \mathrm{~mm}$ when using a 3-point registration on the Accuracy Phantom. Use of a 5-point registration resulted in TRE between 1.3 and $4.6 \mathrm{~mm}$ (fig. 5). The mean error across all 15 intracranial fiducial targets for both experiments was $3.1 \mathrm{~mm}$. The uncertainty in localizing each target ranged from 0.12 to $0.59 \mathrm{~mm}$.

\section{Bench Level Testing}

The test operator selected the adjusted twist drill hole at $10 \mathrm{~cm}$ posterior to the nasion and $5 \mathrm{~cm}$ lateral to the midline on the left frontal approach. The operator successfully targeted the ipsilateral frontal horn of the Procedure Phantom using Smart Stylet guidance in 10 (100\%) attempts from the left frontal approach and $10(100 \%)$ attempts from the right frontal approach. Without image guidance, 4 (40\%) attempts were successful when targeting the right frontal horn and $6(60 \%)$ were unintention-

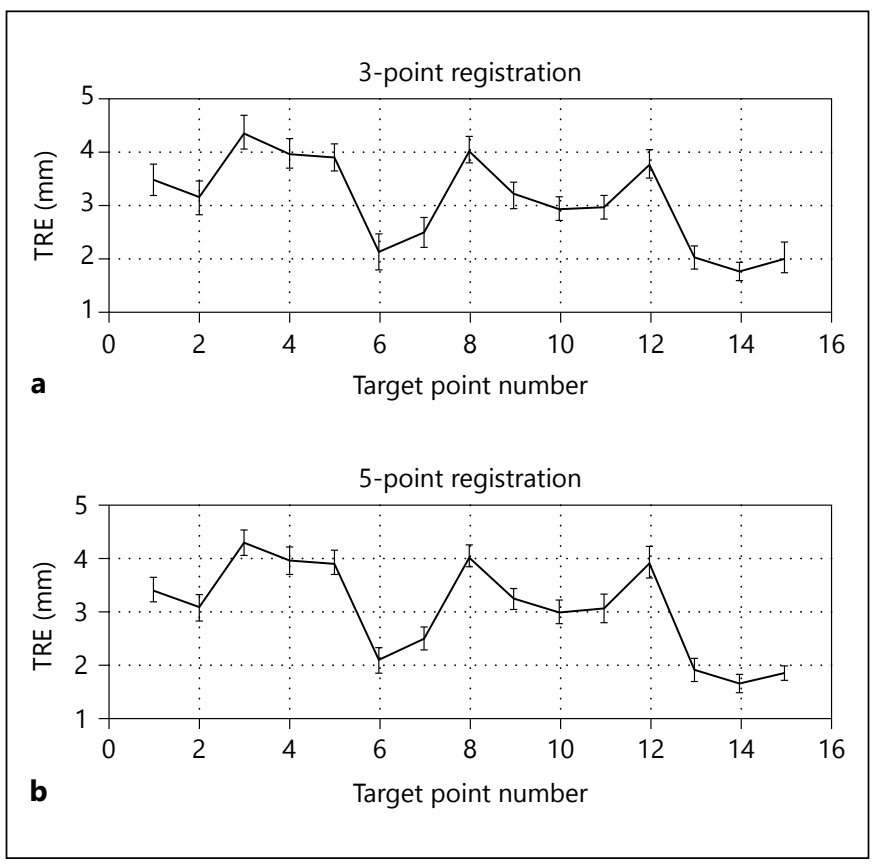

Fig. 5. TRE with five acquisitions of data across 15 intracranial targets in a 3-point registration (a) and 5-point registration (b).

ally targeted to the contralateral frontal horn. From the left frontal approach without image guidance, $6(60 \%)$ attempts were successfully targeted to the ipsilateral frontal horn and $4(40 \%)$ unintentionally to the contralateral frontal horn (table 1).

\section{User Evaluation}

Both residents selected twist drill hole coordinates at $10 \mathrm{~cm}$ posterior to the nasion and $3 \mathrm{~cm}$ lateral to the midline on the left frontal approach. With image guidance from the left frontal approach, one resident successfully targeted the ipsilateral frontal horn and the other resident missed the model. Without Smart Stylet guidance, both residents missed the ventricular model. From the right frontal approach using Smart Stylet guidance, one resident successfully targeted the ipsilateral frontal horn and the other resident unintentionally targeted the contralateral frontal horn of the Procedure Phantom. Without image guidance from the right frontal approach, one resident unintentionally targeted the contralateral frontal horn and the other resident missed the ventricle model (table 1).

\section{Typical Use Scenario}

Smart Stylet in its current developmental stage is a low-cost, rapidly implemented, frameless EM imageguidance system. The prototype computer-assisted sys-
Patil et al. 
Table 1. Targeting results

Hit Contra- Miss lateral

\begin{tabular}{llrll}
\hline Bench level testing & & & \\
Right & with guidance & 10 & 0 & 0 \\
& freehand & 4 & 6 & 0 \\
\hline Left & with guidance & 10 & 0 & 0 \\
& freehand & 6 & 4 & 0 \\
\hline
\end{tabular}

User evaluation

\begin{tabular}{lllll} 
Right & with guidance & 1 & 1 & 0 \\
& freehand & 0 & 1 & 1 \\
\hline Left & with guidance & 1 & 0 & 1 \\
& freehand & 0 & 0 & 2 \\
\hline
\end{tabular}

Bench and User experiments by right and left frontal approaches, with (Smart Stylet) guidance, and freehand. Hit = Success targeting ipsilateral frontal horn; Contralateral = target the contralateral ventricle; Miss $=$ fail to encounter ventricle model.

tem provides advantages over others commercially available for reasons including portability, minimal interference in the EM field from surrounding ferromagnetic objects, real-time tracking, and an intuitive display for the operator. The display was custom-built for targeting during the ventriculostomy procedure. Using the results of a series of proof-of-concept, prototyping and laboratory experiments, we present here a typical use scenario. The Smart Stylet components would be on a small mobile cart that is easily placed at the patient bedside. The use of Smart Stylet does not require fiducial-based registration and so an additional imaging study is not required after the initial screening examination. These volumetric CT images can be transferred from the CT control room computer to the Smart Stylet computer via a universal serial bus (USB) portable disk drive or over the radiology imaging network (data transfer $<30 \mathrm{~s}$ ) to decrease system setup time. A 'metal immune' EM transmitter board is first slipped underneath the head, the head is nonrigidly fixed with foam pads, the Smart Stylet system is calibrated, and the external ventricular drainage (EVD) catheter position can be measured in relation to the patient's head. The EVD cannula is then placed under Smart Stylet guidance, the guidance equipment is removed, and the patient is ready to be sent for a confirmatory CT imaging study. We estimate that this preliminary practice outline would add no more than $10 \mathrm{~min}$ to procedure time.

Smart Stylet: Image Guidance for Bedside Ventriculostomy

\section{Discussion}

The experiments presented in this article were designed to assess the accuracy and utility of Smart Stylet. All components were off-the-shelf including the personal computer, EM tracking system, EVD catheter, and stylet. Once the system was assembled, we determined the sources of system and operator error. We addressed major obstacles when using the prototype Smart Stylet system in environments limited by time, location, and cost.

Custom phantoms were built and each step of the image-registration process analyzed to help isolate system and operator errors. Errors due to system design and setup were more evident when using the Accuracy Phantom in the Accuracy experiment. Placement of the tracked pointer on a visualized target helped localize sources of error to the processes of calibration, registration, and segmentation. The operator errors while carrying out the procedure were best observed while using the Procedure Phantom that housed a shifted ventricular model in the Bench and User experiments.

Smart Stylet was built for implementation without extensive procedure planning and hardware setup. It is not clear how best to quantify intracranial distortions in the event a significant midline shift is not present. One study defined quantitative measures of hydrocephalus using patient measurements derived from CT imaging studies. The article describes that the measure correlates well with patients experiencing hydrocephalus but not with inaccuracies of cannula placement [5]. A phantom with a ventricular system normal in size, morphology, and location would assess an individual's ability to improve on traditional practice. EVDs have traditionally been placed with high levels of accuracy, albeit variable. While preparing the phantom for Bench level testing and User evaluation experiments, fluid or gelatin was not used within the cranial vault to simulate brain parenchyma. It could be argued that selection of a trajectory prior to passing the catheter is commonly the most difficult portion of the procedure. Alternatively, maintenance of a linear trajectory in the Procedure Phantom's air-filled space may prove more technically difficult than navigating through simulated parenchyma. Once in the air-filled cranial vault, the catheter resides in a less constrained environment thus granting the operator increased freedom to deviate from the previously intended linear trajectory. A phantom with simulated parenchyma would require significant adjustments between experiments to eliminate catheter tracts in simulated parenchyma. Using the air-filled phantom, multiple experiments and passes of the Smart Stylet were possible without 
disassembly of the phantom. This method prevented the use of multiple registration matrices, minimized sources of error, and allowed for ease of comparison between trials. We selected a registration method suitable for use at the bedside in the Bench and User experiments by calculating a matrix from a trace of the phantom's calvarium using an EM sensor [18]. In clinical practice, this registration algorithm would minimize time for system setup by removing the requirement to place fiducials followed by an additional preprocedure imaging study.

Results of the Accuracy measurement experiment report an average error of $3.1 \mathrm{~mm}$ when targeting intracranially with Smart Stylet. Bench level testing demonstrated an improved ability for the operator experienced with the system to target a shifted ventricle model in a skull phantom. Similar levels of placement accuracy were observed during the Bench experiment when the operator used the left and right frontal approaches. The targeting accuracy of the residents during the User evaluation experiment was improved with the help of Smart Stylet guidance although the results are not conclusive due to the small sample size. The residents had never interacted with Smart Stylet prior to the experimental attempts. They were given a verbal description of the display without extensive time to study the interface and subsequently requested to pass the catheter. The $50 \%$ success rate using Smart Stylet guidance, while not exemplary, shows an improvement from $0 \%$ and presents the potential for improvement in a technically difficult scenario or in the presence of a midline shift. These preliminary results warrant further development of Smart Stylet and indicate that it may have a place in neurosurgical training and practice.

TRE was calculated to analyze results of the Accuracy measurement experiment and establish a baseline expectation for system error when using Smart Stylet. The accuracy numbers in this article differ from those occasionally used to describe the accuracy of EM systems. Some reports describe manual measurements of system accuracy to confirm targeting information presented to the neurosurgeon $[19,20]$. Other articles report a measure known as the fiducial registration error [21, 22]. The Euclidean distance is calculated between the points representative of fiducials in the CT images and the coordinates from the calibrated, registered pointer placed on the corresponding fiducials in transmitter space. Error measures can be given for each fiducial, averaged to calculate fiducial registration error, and used to assess the quality of a given registration. TRE can then be used to assess how well the operator can target a nonfiducial target point in transmitter space. These calculations become impossible intraoperatively due to fiducial displacement with scalp retraction and target displacement with brain shift. Irrespective of the method used to calculate error margins, baseline error for Smart Stylet using TRE in the Accuracy experiment is comparable to those figures reported in other studies of frameless EM systems [19, 20, 22-24]. During Bench and User experiments, we broadly defined our targeting ability by visualizing the trajectory of the Smart Stylet in relation to the shifted ventricular model. A binary definition of accuracy on pass of the catheter as 'hit' or 'miss' is more relevant than calculated error in a clinical simulation and setting.

Modern optical and EM navigation systems are used exclusively in the operating room. Time-consuming practices that limit use of these systems include the transfer of large imaging data sets from the radiology information systems to the navigation system, segmentation, and registration of the patient to the image. While these tasks may take minutes, the estimated window of opportunity for system deployment is $10 \mathrm{~min}$ [1]. The time to transfer imaging data could be improved by use of a portable USB drive to transfer studies from the CT control room PC to the navigation PC. If the navigation PC is added to the radiology imaging network, images could transfer within seconds. Rapid file transfers could save the neurosurgeon significant amounts of time while deploying the system. Quicker methods of creating three-dimensional visualizations such as volume rendering may be more practical than segmentation. In order to use a portable tracking system such as Smart Stylet at the bedside, registration of the patient to the segmented model must be mastered or further automated. Improvements to the efficiency of system deployment are relevant in emergency clinical scenarios like trauma or other acute presentations of increased intracranial pressure that require rapid intervention.

In its current developmental stage, Smart Stylet differs from modern systems meant for the operating room due to its portable components and unique imaging display. EM sensors are normally placed at convenient locations on instruments meant for the operating room based on sensor size. The distance to the tool tip must then be calculated to accurately measure and display tip position in relation to preprocedure imaging. Calibrating for longer distances results in greater error than when the sensor is placed close to the instrument tip. If the sensor is placed at the base of the catheter stylet, any bends in the catheter due to its weight under gravity and/or during the catheter pass will also cause inaccuracies in the initial calibration. Smart Stylet was designed to minimize these sources of DOI: $10.1159 / 000368906$
Patil et al. 
error by placement of the prototype $0.3-\mathrm{mm}$ EM sensor at the tip of the cannula. The system's tracking functionalities allow the operator to view the EVD catheter and stylet with respect to the segmented preprocedure imaging in real-time. The simulation provides a virtual-reality view of the procedure from the head of the patient's bed. The two oblique views provide more detailed visual feedback to align the trajectory with the intended target in comparison to the information provided in the segmented view.

The portable prototype EM transmitter (fig. 2) was designed for use at the bedside in a trauma bay, emergency room, intensive care unit (ICU) or other inpatient setting. The base is composed of a proprietary material designed by Ascension Technologies that shields the EM field from any ferromagnetic interference below the patient (i.e., hospital gurney and ICU bed frames). The three EM field generators surrounding the patient head were strategically placed to allow for intraprocedural positioning of the patient head. A dynamic frame can be used to compensate for any movement of the patient within the transmitter space $[21,25,26]$. We did not assess any changes to system accuracy due to dynamic frame calculations in this series of experiments. The transmitter design allows for nonrigid fixation or immobilization of the patient head by padding the spaces between the three transmitter bases and the patient. Further studies are being developed to improve Smart Stylet's design and test it within a clinical practice setting.

\section{Study Limitations}

Results of the current study were concluded using phantom simulations and do not directly correlate to clinical practice. Measurements that can be optimized us- ing high precision data capture (calibration, registration and segmentation) required user input. Errors due to calibration and registration can be readily quantified while segmentation errors are more subjective given the large amount of user input necessary to build the three-dimensional image models. TRE was minimized to an average of $3.1 \mathrm{~mm}$. The sensor was not fixed within the catheter and this may have inadvertently caused variability in measurements of catheter position. Operator and resident performance may have been affected by use of a flexible catheter and stylet with an air-filled phantom. Lack of simulated brain parenchyma prevents tactile feedback to the operator and subjects the catheter to gravity. This study may not generalize well to other practitioners given the low number of study participants.

\section{Conclusion}

Smart Stylet provided an advantage over the freehand frontal technique when targeting the ventricles in a phantom experiment representative of distorted ventricular anatomy. The results of this study warrant further development of the prototype Smart Stylet system.

\section{Acknowledgment}

This article is based on a thesis submitted in partial fulfillment of the requirements for the degree of Master of Science in the Harvard-MIT Division of Health Sciences and Technology at the Massachusetts Institute of Technology.

Funding: NIH, R42 CA115112-03, Kirby G. Vosburgh, PhD; NIH, T15 M007092-19, Alexa T. McCray, PhD; Center for Integration of Medicine and Innovative Technology (CIMIT), Boston, Mass., USA.

\section{References}

$>1$ O’Neill BR, Velez DA, Braxton EE, Whiting D, Oh MY: A survey of ventriculostomy and intracranial pressure monitor placement practices. Surg Neurol 2008;70:268-273.

$>2$ O’Leary ST, Kole MK, Hoover DA, Hysell SE, Thomas A, Shaffrey CI: Efficacy of the Ghajar Guide revisited: a prospective study. J Neurosurg 2000;92:801-803.

$>3$ Bogdahn U, Lau W, Hassel W, Gunreben G, Mertens HG, Brawanski A: Continuous-pressure controlled, external ventricular drainage for treatment of acute hydrocephalus - evaluation of risk factors. Neurosurgery 1992;31:898904.

4 Ehtisham A, Taylor S, Bayless L, Klein MW, Janzen JM: Placement of external ventricular

Smart Stylet: Image Guidance for Bedside

Ventriculostomy drains and intracranial pressure monitors by neurointensivists. Neurocrit Care 2009;10: 241-247.

5 Huyette DR, Turnbow BJ, Kaufman C, Vaslow DF, Whiting BB, Oh MY: Accuracy of the freehand pass technique for ventriculostomy catheter placement: retrospective assessment using computed tomography scans. J Neurosurg 2008;108:88-91.

6 Kakarla UK, Kim LJ, Chang SW, Theodore N, Spetzler RF: Safety and accuracy of bedside external ventricular drain placement. Neurosurgery 2008;63:ONS162-ONS167.

-7 Khan SH, Kureshi IU, Mulgrew T, Ho SY, Onyiuke HC: Comparison of percutaneous ventriculostomies and intraparenchymal monitor: a retrospective evaluation of 156 patients. Acta Neurochir Suppl 1998;71:5052.

$>8$ Ngo QN, Ranger A, Singh RN, Kornecki A, Seabrook JA, Fraser DD: External ventricular drains in pediatric patients. Pediatr Crit Care Med 2009; 10:346-351.

$>9$ Saladino A, White JB, Wijdicks EF, Lanzino G: Malplacement of ventricular catheters by neurosurgeons: a single institution experience. Neurocrit Care 2009;10:248-252.

$>10$ Toma AK, Camp S, Watkins LD, Grieve J, Kitchen ND: External ventricular drain insertion accuracy: is there a need for change in practice? Neurosurgery 2009;65:11971201. 
11 Tuli S, O'Hayon B, Drake J, Clarke M, Kestle $\mathrm{J}$ : Change in ventricular size and effect of ventricular catheter placement in pediatric patients with shunted hydrocephalus. Neurosurgery 1999;45:1329-1335.

12 Greenberg M (ed): Handbook of Neurosurgery, ed 7. New York, Thieme, 2010, p 208.

13 Roberts DW: Is good good enough? Neurocrit Care 2009;10:155-156.

14 Walker ML: History of ventriculostomy. Neurosurg Clin N Am 2001;12:101-110.

15 Arabi Y, Memish ZA, Balkhy HH, Francis C, Ferayan A, Al Shimemeri A, Almuneef MA: Ventriculostomy-associated infections: incidence and risk factors. Am J Infect Control 2005;33:137-143.

16 Cleary K, Zhang H, Glossop N, Levy E, Wood $\mathrm{B}$, Banovac F: Electromagnetic tracking for image-guided abdominal procedures: overall system and technical issues. Conf Proc IEEE Eng Med Biol Soc 2005;7:6748-6753.
17 Arun KS, Huang TS, Blostein SD: Leastsquares fitting of two 3-d point sets. IEEE Trans Pattern Anal Mach Intell 1987;9:698700.

18 Besl PJ, McKay ND: A method for registration of 3-D shapes. IEEE Trans Pattern Anal Mach Intell 1992;14:239-256.

19 Rosenow JM, Sootsman WK: Application accuracy of an electromagnetic field-based image-guided navigation system. Stereotact Funct Neurosurg 2007;85:75-81.

20 Benardete EA, Leonard MA, Weiner HL: Comparison of frameless stereotactic systems: accuracy, precision, and applications. Neurosurgery 2001;49:1409-1416.

21 Hayhurst C, Byrne P, Eldridge PR, Mallucci CL: Application of electromagnetic technology to neuronavigation: a revolution in image-guided neurosurgery. J Neurosurg 2009; 111:1179-1184

22 Mascott CR: Comparison of magnetic tracking and optical tracking by simultaneous use of two independent frameless stereotactic systems. Neurosurgery 2005;57:295-301.
23 Rousu JS, Kohls PE, Kall B, Kelly PJ: Computer-assisted image-guided surgery using the Regulus Navigator. Stud Health Technol Inform 1998;50:103-109.

24 Zaaroor M, Bejerano Y, Weinfeld Z, BenHaim S: Novel magnetic technology for intraoperative intracranial frameless navigation: in vivo and in vitro results. Neurosurgery 2001;48:1100-1108.

25 Suess O, Suess S, Mularski S, Kühn B, Picht T, Hammersen S, Stendel R, Brock M, Kombos $\mathrm{T}$ : Study on the clinical application of pulsed DC magnetic technology for tracking of intraoperative head motion during frameless stereotaxy. Head Face Med 2006;2:10.

26 Rodt T, Koppen G, Lorenz M, Majdani O, Leinung M, Bartling S, Kaminsky J, Krauss JK: Placement of intraventricular catheters using flexible electromagnetic navigation and a dynamic reference frame: a new technique. Stereotact Funct Neurosurg 2007;85:243-248. 